\title{
Searching for the statistically equilibrated systems formed in heavy ion collisions
}

\author{
Al. H. Raduta ${ }^{1,2}$, Ad. R. Raduta ${ }^{1,2}$ \\ ${ }^{1}$ GSI, D-64220 Darmstadt, Germany \\ ${ }^{2}$ NIPNE, RO-76900 Bucharest, Romania
}

\begin{abstract}
Further improvements and refinements are brought to the microcanonical multifragmentation model [Al. H. Raduta and Ad. R. Raduta, Phys. Rev. C 55, 1344 (1997); ibid. 61, 034611 (2000)]. The new version of the model is tested on the recently published experimental data concerning the $\mathrm{Xe}+\mathrm{Sn}$ at $32 \mathrm{MeV} / \mathrm{u}$ and $\mathrm{Gd}+\mathrm{U}$ at $36 \mathrm{MeV} / \mathrm{u}$ reactions. A remarkable good simultaneous reproduction of fragment size observables and kinematic observables is to be noticed. It is shown that the equilibrated source can be unambiguously identified.
\end{abstract}

PACS numbers: 24.10.Pa; 25.70.Pq

\section{INTRODUCTION}

It is known from more than 15 years that violent heavy ion collisions lead to an advanced disassembly of the compound system known under the name of nuclear fragmentation. The asymptotically resulted fragments are the only experimental link with the primordial process. Good agreements between various observables related to these fragments and results of various models assuming uniform population of the system phase space [1, 2, 3] led to the conclusion that a statistically equilibrated nuclear source is at the origin of the fragmentation process. The source size, its excitation energy and its volume are thus quantities which can only be indirectly evaluated by comparisons between experimental data and statistical multifragmentation models predictions. The comparison process is complicated by various quantities polluting the pure statistical signals such as preequilibrium particle emission, collective radial expansion, Coulomb propagation of the break-up fragments, secondary particle emissions. A complete theoretical reproduction of experimental fragmentation event topology (never achieved so far) would help to answer questions fundamental for determining the thermodynamics taking place in such systems. For example, the identification of the experimental freeze-out volume would allow to locate the process in the phase diagram and, therefore, would help solving a problem which made the subject of a strong debate over the last decade: is there any phase transition taking place in nuclear matter?

The aim of the present paper is to investigate whether an unambiguous determination of the experimental statistically equilibrated source can be achieved by means of the microcanonical model from Ref. 4, 5]. To this aim, the model is refined by including the conservation of the angular momentum and completed with a Coulomb propagation stage of the primary fragments with the possibility of superimposing radial flow. A complete set of experimental fragmentation data concerning the reactions $\mathrm{Xe}+\mathrm{Sn}$ at $32 \mathrm{MeV} / \mathrm{u}$ and $\mathrm{Gd}+\mathrm{U}$ at $36 \mathrm{MeV} / \mathrm{u}$ recently published in Ref. 6, 7] is used for comparison with the model results. The paper is structured as follows: Section II gives a brief presentation of the employed microcanonical model and describes its new refinements and improvements. In Section III a comparison between the model prediction and the above mentioned experimental data is presented. The influence of various model parameters on different observables is discussed in Section IV. Conclusions are drawn in Section V.

\section{THE MODEL: BRIEF REVIEW AND IMPROVEMENTS}

The microcanonical multifragmentation model used in the present study was proposed in Ref. [4, 5. The modifications from the previous version of the model concern the primary break-up stage, momentum generation, inclusion of collective radial expansion, Coulomb propagation, and the secondary emission stage.

i) Break-up stage

This stage concerns the disassembly of a statistically equilibrated nuclear source $(A, Z, E, V)$ (the mass number, the atomic number, the excitation energy and the freeze-out volume respectively). The basic assumption of the model is equal probability between all configurations $C:\left\{A_{i}, Z_{i}, \epsilon_{i}, \mathbf{r}_{i}, \mathbf{p}_{i}, \quad i=1, \ldots, N\right\}$ (the mass number, the atomic number, the excitation energy, the position and the momentum of each fragment $i$ of the configuration $C$, composed of $N$ fragments). Fragments are assumed to be spherical, are not allowed to overlap each other and are placed into a spherical recipient of volume $V$. In the previous version of the model [4] the system was considered subject to the standard microcanonical constraints: $\sum_{i} A_{i}=A, \sum_{i} Z_{i}=Z, \sum_{i} \mathbf{p}_{i}=\mathbf{P}(=0$ in the c.m. frame), $E$ - constant. An extra constraint will be considered herein: the conservation of the angular momentum, $\sum_{i} \mathbf{r}_{i} \times \mathbf{p}_{i}=\mathbf{L}$. The integration over fragment momenta can be analytically performed subject to the above mentioned constraints: 


$$
\begin{aligned}
& \int \prod_{i=1}^{N} \mathrm{~d} \mathbf{p}_{i} \delta(H-E) \delta\left(\sum_{i} \mathbf{p}_{i}-\mathbf{P}\right) \delta\left(\sum_{i} \mathbf{r}_{i} \times \mathbf{p}_{i}-\mathbf{L}\right) \\
= & \frac{2 \pi}{\Gamma\left(\frac{3}{2}(N-2)\right)}\left(\frac{\prod_{i} m_{i}}{\sum_{i} m_{i}}\right)^{3 / 2} \frac{1}{\sqrt{\operatorname{det} \mathbf{I}}}\left[2 \pi\left(K-\frac{P^{2}}{2 M}-\frac{1}{2} \mathbf{L}^{T} \mathbf{I}^{-1} \mathbf{L}\right)\right]^{\frac{3}{2}(N-2)-1}
\end{aligned}
$$

Here $M=\sum_{i} m_{i}, \mathbf{I}$ is the inertial tensor of the system: $I_{\alpha \beta}=\sum_{i} m_{i}\left(r_{i}^{2} \delta_{\alpha \beta}-r_{i}^{\alpha} r_{i}^{\beta}\right)$ with $\alpha, \beta=1,2,3$, $\mathbf{L}^{T} \mathbf{I}^{-1} \mathbf{L}=\sum_{\alpha \beta=1}^{3} L_{\alpha} I_{\alpha \beta}^{-1} L_{\beta}, \quad H=\sum_{i} p_{i}^{2} /\left(2 m_{i}\right)+$ $\sum_{i<j} V_{i j}+\sum_{i} \epsilon_{i}-\sum_{i} B_{i}$ and $K=E-\sum_{i<j} V_{i j}-$ $\sum_{i} \epsilon_{i}+\sum_{i} B_{i}$ ( $V_{i j}$ stands for the Coulomb interaction between fragments $i$ and $j$ ). Of course, the model corresponds to the c.m. frame (i.e. $\mathbf{r}_{i}$ with $i=1, \ldots, N$ are positions in the c.m. frame) where $\mathbf{P}=0$ [14]. If one also imposes $\mathbf{L}=0$ (which is the hypothesis employed in the present calculations) then the last factor in eq. (11) becomes $(2 \pi K)^{3 / 2 N-2}$. The only modifications brought by the inclusion of the angular momentum conservation constraint to the statistical weight of a configuration $C^{\prime}:\left\{A_{i}, Z_{i}, \epsilon_{i}, \mathbf{r}_{i}, i=1, \ldots, N\right\}\left(W_{C^{\prime}}\right)$ from Ref. [4] are the inclusion of the factor $1 / \sqrt{\operatorname{det} \mathbf{I}}$ and the replacement of $N$ with $N-1$. These new weights can be employed in a Metropolis-type simulation which allows the determination of the average value of any system observable in the very same manner as in Ref. 䛧.

Fragments with $A \leq 4$ are considered without excitation degrees of freedom except for the $\alpha$ particle for which the few levels larger than $20 \mathrm{MeV}$ with $\Gamma \leq 2.01 \mathrm{MeV}$ have been considered. These fragments are weighted in $W_{C^{\prime}}$ with their energy levels degeneracies. Larger fragments carry internal excitation. For them, the following level density formula is included in the statistical weight of a configuration $C^{\prime}$ (see Ref. [5]):

$$
\rho(\epsilon)=\frac{\sqrt{\pi}}{12 a^{1 / 4} \epsilon^{5 / 4}} \exp (2 \sqrt{a \epsilon}) \exp (-\epsilon / \tau)
$$

with $a=0.114 A+0.098 A^{2 / 3} \mathrm{MeV}^{-1}$ [8] and $\tau=9 \mathrm{MeV}$. The factor $\exp (-\epsilon / \tau)$ is introduced to account for the dramatic decrease of the excited levels lifetime at high excitation energies [3].

ii) Generation of the primary decay fragment momenta While integration over the fragments' momenta has been carried out in order to simplify the Metropolis simulation, to produce events similar to the experimental ones one has to generate momenta for each given configuration $C^{\prime}$. Each of these events is characterized by a kinetic energy $K$. Therefore, we have to deal with the following computational task: generate uniformly momenta for a system composed of $N$ particles such that $\sum_{i} p_{i}^{2} / 2 m_{i}=K$, $\sum_{i} \mathbf{p}_{i}=0$ and $\sum_{i} \mathbf{r}_{i} \times \mathbf{p}_{i}=\mathbf{L}$. This problem was solved in an elegant way in Ref. [9]. There it is proved that the following generation gives the right sampling of the system momenta, in agreement with the above mentioned constraints: Pick a preliminary set of $N$ particle momenta from a arbitrary canonical distribution, then eliminate the overall translational and rotational motion by making the transformation $\mathbf{p}_{i} \rightarrow \mathbf{p}_{i}-m_{i}\left(\mathbf{P}^{\prime} / M+\boldsymbol{\omega}^{\prime} \times \mathbf{r}_{i}\right)$ (where $\mathbf{P}^{\prime}=\sum_{i} \mathbf{p}_{i}$ and $\boldsymbol{\omega}^{\prime}=\sum_{i} \mathbf{r}_{i} \times \mathbf{p}_{i} \cdot \mathbf{I}^{-1}$ ), then spin the system such as to match the desired angular momentum $\left.\mathbf{L}: \mathbf{p}_{i} \rightarrow \mathbf{p}_{i}+m_{i} \boldsymbol{\omega} \times \mathbf{r}_{i}\right)$ with $\boldsymbol{\omega}=\mathbf{L}^{T} \cdot \mathbf{I}^{-1}$ and finally, renormalize the momenta such as to match the available energy $K-\frac{1}{2} \boldsymbol{\omega} \cdot \mathbf{L}$.

\section{iii) Radial flow}

After generating the fragment momenta corresponding to the primary decay, inclusion of nonequilibrium effects such as collective radial expansion (flow) can be easily superimposed. Here we use the following parameterization for the flow velocity of fragment $i: \mathbf{v}_{f}^{i}=\mathbf{v}_{0}\left(r_{i} / R\right)^{\alpha}$, with $\mathbf{v}_{0}=v_{0}\left(\mathbf{r}_{i} / r_{i}\right)$ and $\alpha$ a real number defining the flow profile. After including the radial flow the momentum of the $i$ th fragment reads: $\mathbf{p}_{i} \rightarrow \mathbf{p}_{i}+m_{i} \mathbf{v}_{f}^{i}$.

iv) Coulomb propagation

After break-up, hot primary fragments are supposed to suffer an expansive propagation under their mutual Coulomb interaction. This stage can be easily simulated by integrating the corresponding Newtonian equations of motion. Integration has been carried out up to $500 \mathrm{fm} / \mathrm{c}$, when Coulomb interaction between fragments can be neglected.

\section{v) Secondary decays}

Since primary fragments carry internal excitation, a secondary decay stage was introduced in Ref. [5]. Depending on the fragment excitation, secondary break-up processes or particle evaporation were considered. Since the above classification is rather arbitrary, here we resume to the second process, treated using the standard Weisskopf evaporation scheme. As in [5], the range of the evaporated particles is considered up to $A=16$. Evaporation events are simulated using standard Monte Carlo [5].

For each primary break-up event (i), the steps ii) - v) are performed. The resulted fragmentation events can be readily compared with experimental ones (after removing the particles coming from the preequilibrium stage).

\section{COMPARISON OF THE MODEL PREDICTIONS WITH RECENT EXPERIMENTAL DATA}

In this section comparison between the model results and the recent experimental data concerning the reac- 

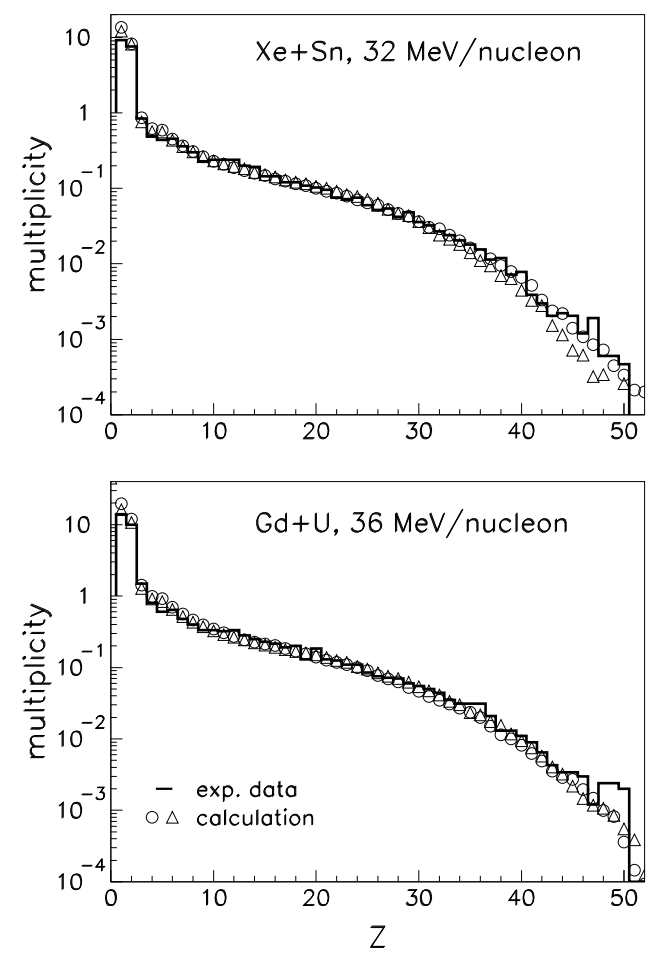

FIG. 1: Calculated charge multiplicities in comparison with experimental data for the $\mathrm{Xe}+\mathrm{Sn}$ at $32 \mathrm{MeV} / \mathrm{u}$ and $\mathrm{Gd}+\mathrm{U}$ at $36 \mathrm{MeV} / \mathrm{u}$ reactions. Open circles correspond to calculation using the freeze-out hypothesis (1), open triangles correspond to calculation using the hypothesis (2). Experimental data are represented by the histogram.

tions $\mathrm{Xe}+\mathrm{Sn}$ at $32 \mathrm{MeV} / \mathrm{u}$ and $\mathrm{Gd}+\mathrm{U}$ at $36 \mathrm{MeV} / \mathrm{u}$ are presented. For the above reactions, various experimental fragment size and kinematic observables have been published recently [6, 7]. The aim of the study is to extract information about the physical phenomenon taking place by trying to fit the entire set of experimental data using the microcanonical multifragmentation model. It is known [10] that various freeze-out assumptions may induce important differences in the statistical models' results. For drastically reducing this uncertainty in the present study we employ two opposite freeze-out scenarios:

1) The standard working hypothesis of the model: Fragments are idealized as hard spheres placed into a spherical freeze-out recipient; fragments are not allowed to overlap each other or the recipient wall. The generation of the fragments' positions is described in Ref. 何.

2) The hardcore interaction is switched off: Integration over the fragments' positions may be approximately carried out by assuming as in Ref. [12 that each fragment (from a configuration composed of $N$ fragments) is blocking the volume $V_{0} / N$ ( $V_{0}$ is the volume of the nuclear system at normal density) for the rest of the fragments (as in 112) and for itself as well. Coulomb energy is approximated by the Wigner Seitz formula, being therefore independent of the fragments' positions [15]. The inte-

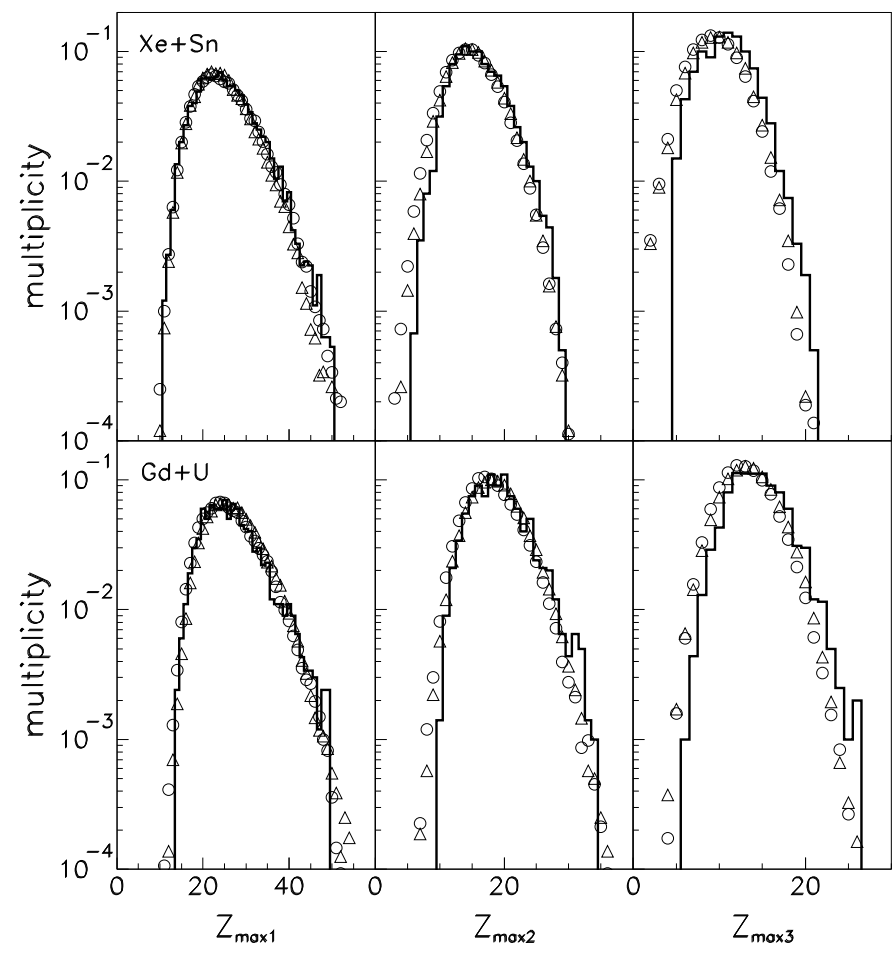

FIG. 2: Calculated distributions of the largest, second largest and third largest charge in one fragmentation event in comparison with the corresponding experimental data for the $\mathrm{Xe}+\mathrm{Sn}$ at $32 \mathrm{MeV} / \mathrm{u}$ and $\mathrm{Gd}+\mathrm{U}$ at $36 \mathrm{MeV} / \mathrm{u}$ reactions. Symbols are used as in Fig. 1.

gration over the fragments' positions writes:

$$
\int \prod_{i=1}^{N} \mathrm{~d} \mathbf{r}_{i} \frac{1}{\sqrt{\operatorname{det} \mathbf{I}}} \prod_{j<i} \theta_{i j} \simeq V_{\text {free }} \int \prod_{i=1}^{N} \mathrm{~d} \mathbf{r}_{i} \frac{1}{\sqrt{\operatorname{det} \mathbf{I}}}
$$

with:

$$
V_{\text {free }}=\prod_{i=1}^{N}\left(V-i \frac{V_{0}}{N}\right) .
$$

The factor $\prod_{j<i} \theta_{i j}$ from eq. (3) is just a formal expression of the fragment blocking constraint formulated before. With this approximation fragment positions have to be generated into the spherical freeze-out volume, without any hardcore constraint only for evaluating $\operatorname{det} \mathbf{I}$ and the statistical weight of a configuration will get (as in Ref. [10]) the extra factor $V_{\text {free }}$. Having the weights of each configuration $C^{\prime}$ the corresponding Metropolis type simulation is straight forward 何.

Using the above defined working hypotheses, comparisons between the model's results and the corresponding experimental data have been performed. Both fragment size distributions and kinematic distributions have been considered. Very good agreement between calculations and experimental data can be observed for both reactions, in both considered working hypotheses for all the considered observables. 

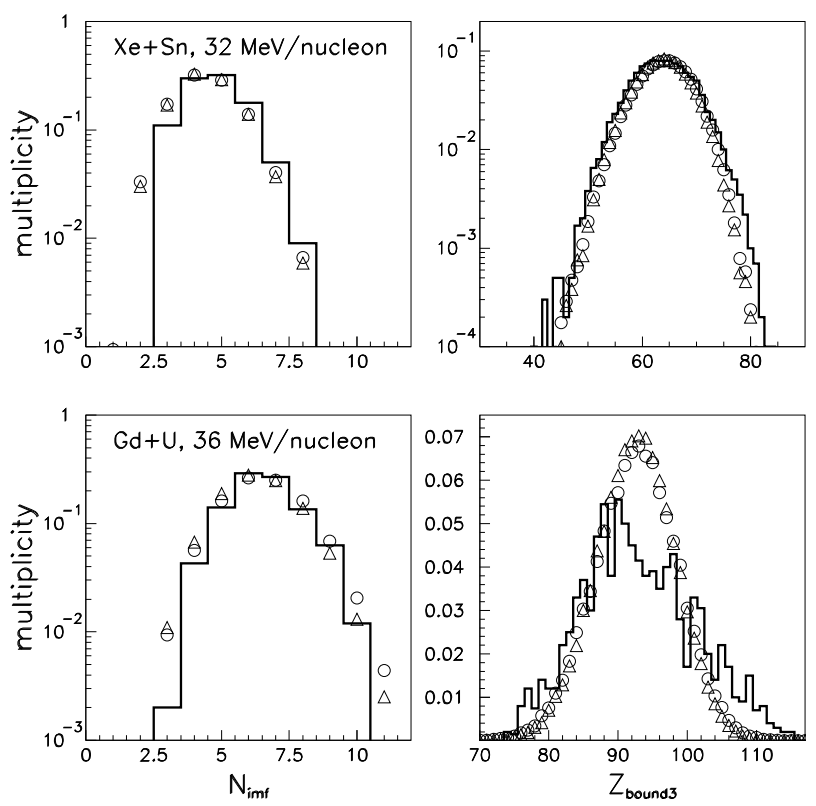

FIG. 3: Calculated IMF multiplicities (left column) and $Z_{\text {bound } 3}$ multiplicities (right column) in comparison with the corresponding experimental data for the reactions $\mathrm{Xe}+\mathrm{Sn}$ at $32 \mathrm{MeV} / \mathrm{u}$ (upper raw) and $\mathrm{Gd}+\mathrm{U}$ at $36 \mathrm{MeV} / \mathrm{u}$ (lower raw). Symbols are as in Fig. 1.

The comparisons between theoretical and experimental fragment size distributions corresponding to both $\mathrm{Xe}+\mathrm{Sn}$ at $32 \mathrm{MeV} / \mathrm{u}$ and $\mathrm{Gd}+\mathrm{U}$ at $36 \mathrm{MeV} / \mathrm{u}$ reactions are as follows: Charge multiplicities are given in Fig. 1. Multiplicities of largest charge $\left(Z_{\max 1}\right)$, second largest charge $\left(Z_{\max 2}\right)$ and third largest charge $\left(Z_{\max 3}\right)$ from one fragmentation event are presented in Fig. 2. In Fig. 3 intermediate mass fragment (IMF) distributions and $Z_{\text {bound } 3}$ distributions are given (IMF fragments are considered to have $Z \geq 5$ as in [7]; $Z_{\text {bound } 3}$ is defined as the sum over all fragments' atomic numbers from one fragmentation event which are greater or equal to $3 ; Z_{\text {bound5 }}$ is similarly defined but the $Z$ limit is fixed to 5 ). $Z_{\text {bound5 }}$ multiplicities are compared with experimental data in Fig. 4 only for the reaction $\mathrm{Gd}+\mathrm{U}$ at $36 \mathrm{MeV} / \mathrm{u}$. It can be noticed that for the $Z_{\text {bound }}$ distributions the calculated width of the distributions are slightly smaller than the experimental ones. This suggests a (small) fluctuation of the experimental equilibrated source. This will become more clear in the next section. An excellent agreement for all considered observables related to fragment size was, therefore, obtained.

Comparisons between theoretical and experimental average fragment kinetic energy function of fragment charge are given in Fig. 5, left column for the the two considered reactions. Very good agreements are to be noticed. A deeper insight to the kinematics of the process can be obtained by analyzing the reduced fragments velocity correlations [11. Fragments with $5 \leq Z \leq 20$ as in 13] have been used for constructing the correlations. This observable was proven to reflect subtle topological features of the fragmentation events. The beginning of the correlation function is often called "Coulomb hole" and reflects the strength of the Coulomb repulsion between fragments (obviously related to the size of the freezeout volume) but, as shown in the next section, is also related to the amount of radial expansion. Good agreement between calculations and experimental data can be observed here as well (see Fig. 5). While in the case of the $\mathrm{Gd}+\mathrm{U}$ reaction the experimental velocity correlations are well reproduced in both freeze-out hypotheses, in the case of the first reaction a better agreement with experimental data is obtained for the hypothesis (2) ("without hard-core"). In fact, this result is natural and suggests that deviations from the spherical fragments with hard-core interaction idealization (deformed fragments, surface diffusivity, etc.) are present in real multifragmentation. The deviation effect is stronger in the case of the $\mathrm{Xe}+\mathrm{Sn}$ since the corresponding equilibrated system is smaller and, therefore the fragment partitions are more symmetric (as shown in [10], the free volume is smaller for more symmetric partitions and larger for more asymmetric ones). The simultaneous description of both average fragment kinetic energies as a function of the fragment charge and of the reduced velocity correlations is remarkable and reflects a good description of the event topology. Here it is worth noticing that in Ref. [13] the velocity correlations for both reactions could not be reproduced via the SMM model.

The values of the model input parameters for which the comparisons with the experimental data have been performed are listed in Table II. A remarkable fact is that the fitting parameters corresponding to the freezeout hypotheses (1) and (2) are quite close ones to the others for each of the considered reactions. This tends to indicate a relatively small "region", specific to each reaction in which the parameters of the real equilibrated nuclear source are situated. Quite small differences from one hypothesis to the other seem to be present in the case of the freeze-out volume. This result may look intriguing given the fact that freeze-out volume is directly related

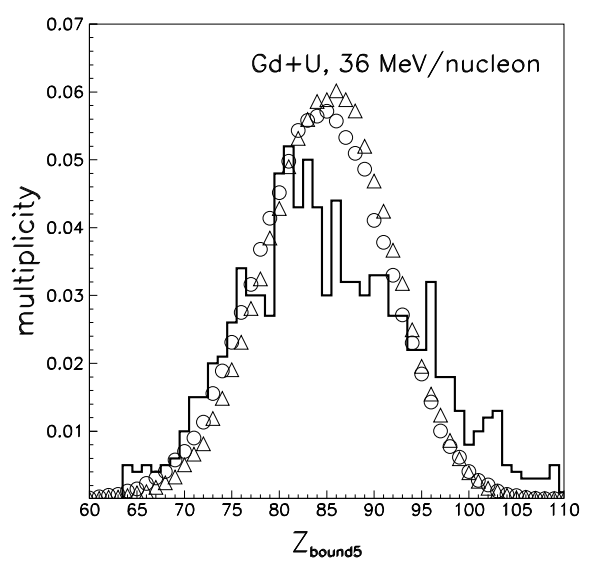

FIG. 4: Same as in Fig. 3 (right column) for the $Z_{\text {bound5 }}$ distribution corresponding to the $\mathrm{Gd}+\mathrm{U}$ at $36 \mathrm{MeV} / \mathrm{u}$ reaction. 

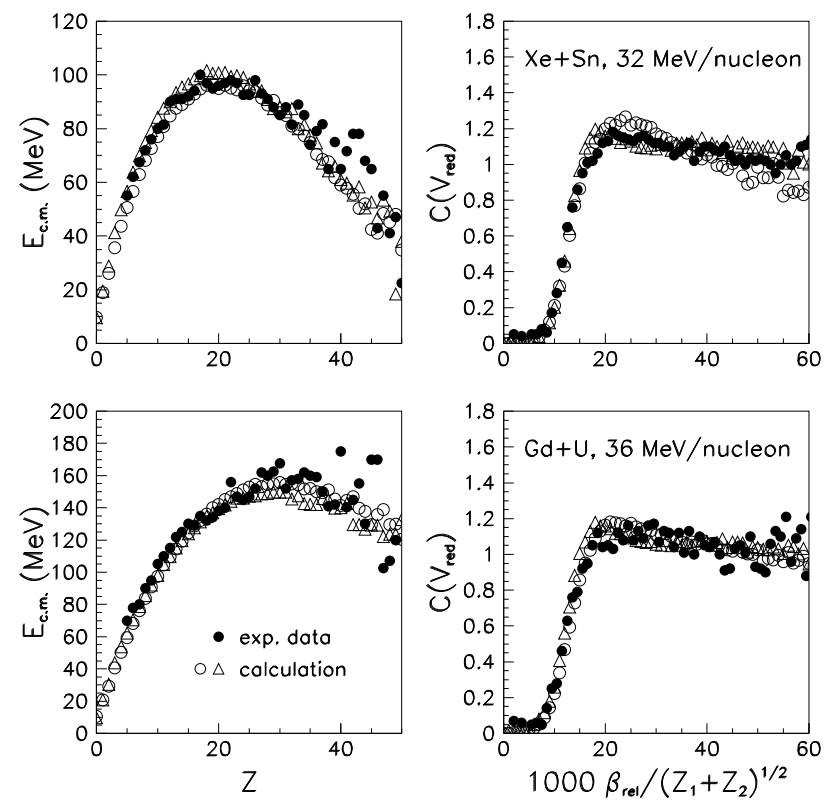

FIG. 5: Average fragment kinetic energy versus charge (left column) and reduced fragment velocity correlation (right column) for the reactions $\mathrm{Xe}+\mathrm{Sn}$ at $32 \mathrm{MeV} / \mathrm{u}$ (upper raw) and $\mathrm{Gd}+\mathrm{U}$ at $36 \mathrm{MeV} / \mathrm{u}$ (lower raw). Symbols are as in Fig. 1 except for the experimental data which are here represented by full circles.

to both working hypotheses. In reality, the relatively large freeze-out volumes obtained are minimizing the influence of the hard-core interactions to the free-volume $\left(V_{\text {free }}\right)$ (see e.g. [10]), the values of of the freeze-out volume being mainly dictated by the Coulomb interaction (the remaining quantity entering the system's density of states depending on the freeze-out volume). Unlike the free volume which in models can only be more or less arbitrary parametrized, Coulomb interaction can be accurately evaluated for each fragment partition (either by

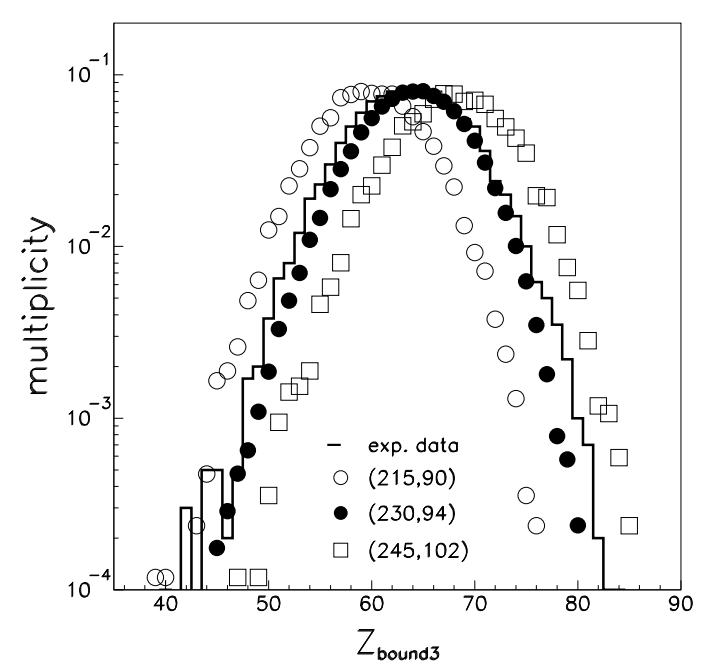

FIG. 6: $Z_{\text {bound3 }}$ distributions (symbols) corresponding to various sizes of the nuclear source within the working hypothesis (1) (see the legend).
TABLE I: Values of the model's input parameters corresponding to the fitting of the experimental data for both $\mathrm{Xe}+\mathrm{Sn}$ at $32 \mathrm{MeV} / \mathrm{u}$ and $\mathrm{Gd}+\mathrm{U}$ at $36 \mathrm{MeV} / \mathrm{u}$ reactions in the freeze-out hypotheses (1) and (2). The last two columns correspond respectively to the flow energy and to the exponent defining the flow profile (see text).

\begin{tabular}{cccccc}
\hline \hline react./hyp. & $(A, Z)$ & $V / V_{0}$ & $E_{e x}(\mathrm{MeV} / \mathrm{u})$ & $E_{f l}(\mathrm{MeV} / \mathrm{u})$ & $\alpha$ \\
\hline Xe+Sn $(1)$ & $(230,94)$ & 9 & 5.3 & 1.4 & 2 \\
Xe+Sn $(2)$ & $(220,92)$ & 8.5 & 4.78 & 1.9 & 1.8 \\
\hline Gd+U (1) & $(343,136)$ & 8.5 & 5.95 & 1.7 & 2 \\
Gd+U (2) & $(328,130)$ & 8 & 5.2 & 1.9 & 1.8 \\
\hline \hline
\end{tabular}

explicitly accounting for the interfragment interaction, or by employing the Wigner-Seitz approach). This contributes to the solidity of the present evaluations.

The differences in the excitation energies corresponding to the two hypotheses are due to the Coulomb energy variation from one case to another, caused by the slightly different values of the freeze-out volumes (see Table (i). For example, in the $\mathrm{Gd}+\mathrm{U}$ case the energy variation (from (1) to (2)) is slightly larger than in the $\mathrm{Xe}+\mathrm{Sn}$ case since the relative variation of the freeze out volume is larger as well. Freeze-out volume is always smaller in the working hypothesis (2). This is related to the different dependencies $V_{\text {free }}(V)$ resulting from the underlying hypotheses (more symmetric partitions and thus a more advanced fragmentation are specific to the without hardcore case and therefore both the freeze-out volume and excitation energies need to be smaller in the second hypothesis). The flow parameters were chosen such as to insure a good reproduction of the kinetic observables (see Fig. 5). The way in which each parameter is influencing the fitting observables is discussed in the next section.

\section{INFLUENCE OF THE MODEL PARAMETERS ON VARIOUS FITTING OBSERVABLES}

While many fragment size and kinetic observables were simultaneously fitted in order to deduce the parameters of the equilibrated sources corresponding to the two reactions, the following question arises: are they unique? We address this question in the present section.

We start with the remark that the size of the equilibrated source appears to be dictated by the $Z_{\text {bound }}$ distribution. We illustrate this by representing in Fig. 6 the $Z_{\text {bound } 3}$ distributions corresponding to three different sources: $(215,90),(230,94)$ and $(245,102)$. Their excitation energies $(5.4,5.3,5.3 \mathrm{MeV} / \mathrm{u}$ respectively) are chosen such as to insure a good fit of the charge distributions corresponding to the $\mathrm{Xe}+\mathrm{Sn}$ at $32 \mathrm{MeV} / \mathrm{u}$ reaction; their freeze-out volume $\left(V / V_{0}=9\right)$ is chosen as to provide a good description of the $Z_{\max }, Z_{\max 2}$ and $Z_{\max 3}$ distributions. It can be easily observed that the best fit 


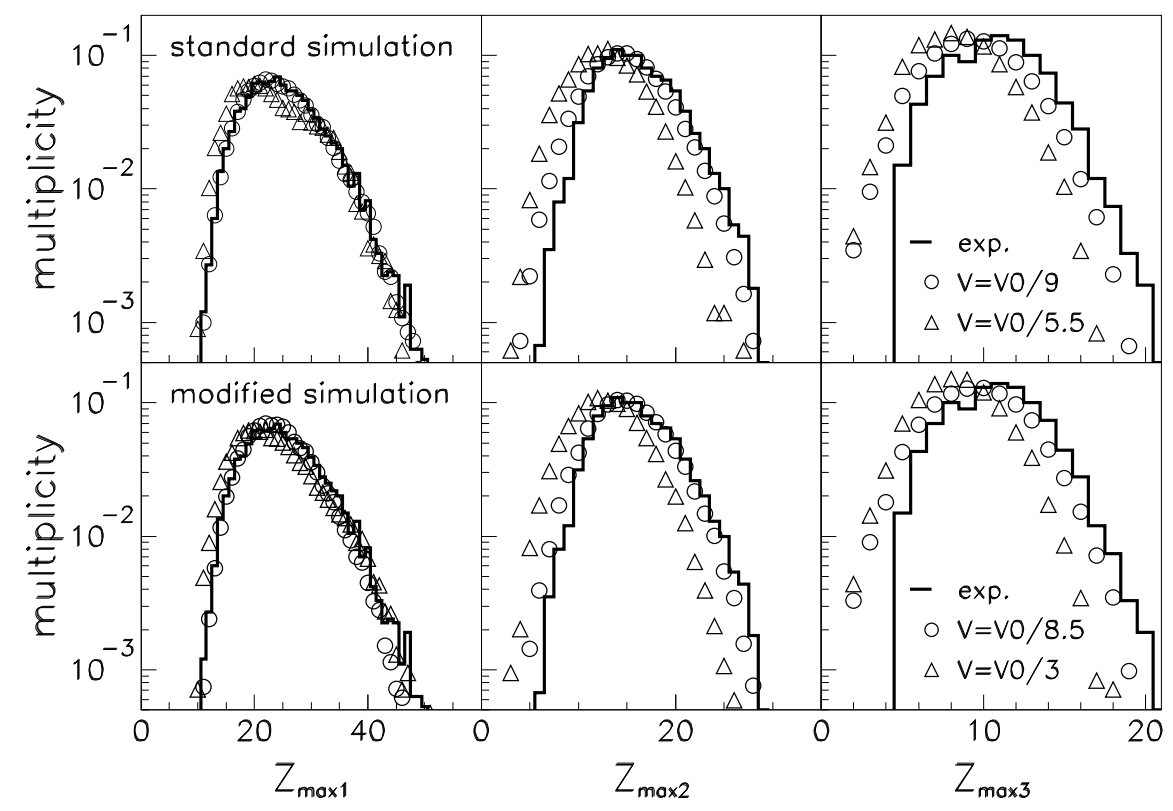

FIG. 7: Largest, second largest and third largest charge distributions corresponding to two different freeze-out volumes (see the legend) for each of the two freeze-out hypotheses ((1) upper raw; (2) lower raw) in comparison with Xe+Sn at 32 MeV/u the experimental data. See the text for the sources' parameters.

on the data is given by the $(230,94)$ source. A smaller source deviates the maximum of the $Z_{\text {bound } 3}$ distribution to the left while a larger one deviates it to the right (see Fig. 6).

The freeze-out volume of the equilibrated sources appears to be dictated by the distributions of the largest, second largest and third largest charge in one fragmentation event. Obviously, these distributions provide a measure of the degree of asymmetry of a given fragment partition. The degree of asymmetry of a partition is influenced by the freeze-out volume through both the Coulomb interaction and the free volume [10]. This fact is evidenced in Fig. 7 where multiplicities of $Z_{\max 1}, Z_{\max 2}$ and $Z_{\max 3}$ are represented for two different freeze out volumes in the freeze-out hypotheses (1) and (2) as follows. For the first hypothesis the source was considered $(230,94)$ at two different volumes: $V_{0} / 5.5$ and $V_{0} / 9$ while for the second one the source was taken to be $(220,92)$ at other two different volumes $V_{0} / 3$ and $V_{0} / 8.5$. The corresponding excitation energies are taken such as a good reproduction of the experimental charge distributions corresponding to the $\mathrm{Xe}+\mathrm{Sn}$ reaction at $32 \mathrm{MeV} / \mathrm{u}$ to be achieved (the excitations energies are respectively: $6.4,5.3,6.1,4.78 \mathrm{MeV} / \mathrm{u}$ ). It can be noticed that deviations to the left of the maxima (indicating more asymmetric fragment partitions) of the $Z_{\max 2}$ and $Z_{\max 3}$ distributions are occurring when the freeze-out volume is decreased. This behavior can be observed irrespective to the considered freeze-out hypothesis or to the chosen mass of the source. While, as demonstrated by Fig. 7 lower values of the freeze-out volume are leading to deviations of the $Z_{\max 1}, Z_{\max 2}$ and $Z_{\text {max } 3}$ calculated distributions from the corresponding experimental data, larger values of the freeze-out volume will result in smaller excitation energies and larger flow energies leading to deviations from the experimental data of the kinematic observables represented in Fig. 5.

Given the previously described monotonical behavior of the "distance" from the calculated curves to the ex-
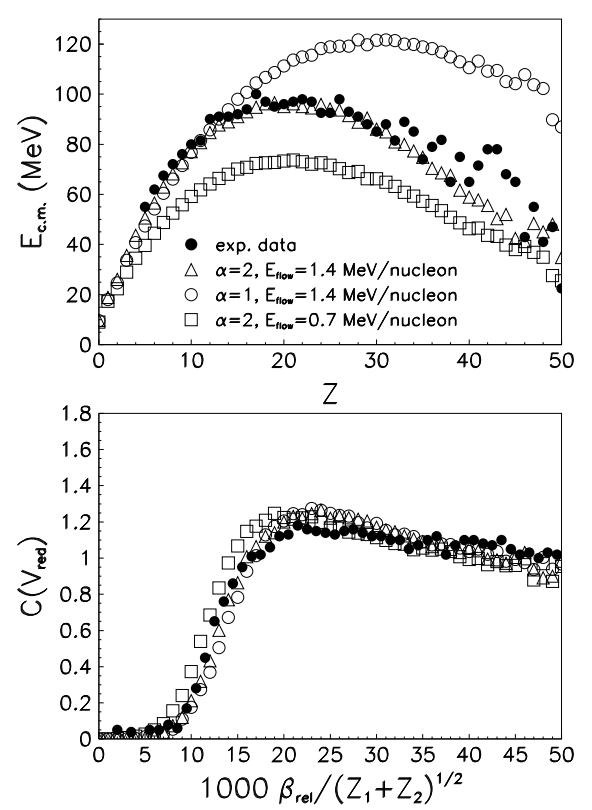

FIG. 8: Influence of the radial flow parameters on the fragment average kinetic energy versus charge dependency and on the fragment reduced velocity correlations for the working hypothesis (1) (see the legend and the text for the sources' parameters). The experimental data are here represented by full circles. 
perimental ones, and also the very good reproduction of all the considered experimental observables for the parameters (given in Table I) corresponding to the case in which the $Z_{\text {bound } 3}$ distribution is fitted best (see Fig. 6) it results that the corresponding sets of parameters provide the global minimum of the associated $\chi^{2}$ function. Indeed, choosing a different value of $A$ would generate a deviation of the $Z_{\text {bound3 }}$ from the experimental one and, obviously the global reproduction of the data would be worst.

Finally, the way in which the radial flow parameters $E_{f l}$ and $\alpha$ are influencing the experimental data fitting is presented in Fig. 8 for the nuclear source $(230,94)$, $V=V_{0} / 9, E_{e x}=5.3 \mathrm{MeV} / \mathrm{u}$ and the first working freezeout hypothesis. Three different cases are being considered: $\left(\alpha=2, E_{f l}=1.4 \mathrm{MeV} / \mathrm{u}\right),\left(\alpha=1, E_{f l}=1.4\right.$ $\mathrm{MeV} / \mathrm{u})$ and $\left(\alpha=2, E_{f l}=0.7 \mathrm{MeV} / \mathrm{u}\right)$. As shown in Fig. 8 the $\alpha$ parameter defining the flow profile appears to influence the location of the maximum of the average kinetic energy versus $Z\left(E_{c . m .}(Z)\right)$ and the value of the flow energy the height of the $E_{c . m .}(Z)$ dependency. Smaller effects are to be noticed in the reduced velocity correlation function and they concern mainly the width of the "Coulomb hole". The effects are intuitive: smaller flow leads to a narrower Coulomb hole while larger ones lead to a larger hole. The parameters $\alpha=2$ and $E_{f l}=1.4 \mathrm{MeV} / \mathrm{u}$ appear to give the best description of the kinetic experimental data corresponding to the $\mathrm{Xe}+\mathrm{Sn}$ at $36 \mathrm{MeV} / \mathrm{u}$ reaction.

Therefore, it results that, within some (small) inherent uncertainties, the obtained parameters corresponding to the two working hypotheses ((1) and (2)) are uniquely determined.

\section{CONCLUSIONS}

In summary, the microcanonical multifragmentation model [4] has been further refined and improved. Conservation of angular momentum has been included. Also, in order to provide events similar with the experimental ones, the model is completed with a stage of generation of momenta for the primary break-up fragments. Radial flow velocities can be superimposed here. Finally, an expansion stage of the hot primary fragments under their mutual Coulomb interaction is added. Using the new version of the model, the recent experimental data of the INDRA collaboration concerning the reactions
$\mathrm{Xe}+\mathrm{Sn}$ at $32 \mathrm{MeV} / \mathrm{u}$ and $\mathrm{Gd}+\mathrm{U}$ at $36 \mathrm{MeV} / \mathrm{u}$ are analyzed. Both fragment size distribution observables and kinematic observables could be simultaneously fitted allowing thus the identification of the equilibrated sources parameters corresponding to the two reactions. Two different break-up scenarios were used for performing the analysis. The two types of calculations give close results for each of the considered reactions suggesting independence of the obtained results on the particular hypothesis. This is due to the fact that for relatively large values of the freeze-out volume (such as those obtained by fitting the data, 8-9 $V_{0}$ ) the influence of the hard-core is small and thus the results are rather independent on the extent to which these effects are included in a specific $V_{\text {free }}$ parametrization. In this case, the values of the freeze-out volume are dictated by the Coulomb interaction (accurately evaluated independently to any model assumptions) which makes the evaluation particularly robust. For each reaction a narrow freeze-out volume region was identified (8.5-9 $V_{0}$ for $\mathrm{Xe}+\mathrm{Sn}$ and 8-8.5 $V_{0}$ for $\left.\mathrm{Gd}+\mathrm{U}\right)$. By analyzing the way in which variations of the model parameters are influencing the deviation between the calculated curves and the experimental ones, it is proved that the set of the parameters providing the best fit to the data is unique (within inherent uncertainties). It is shown that the size of the source is dictated by the $Z_{\text {bound }}$ distributions while its freeze-out radius can be deduced by fitting the experimental distributions of $Z_{\max 1}, Z_{\max 2}$ and $Z_{\max 3}$ and the kinematic observables. The way in which the flow energy and the parameter $\alpha$, defining the flow profile is influencing the fragment average kinetic energy versus $Z$ and also the reduced velocity correlation is also discussed. The above parameters are shown to be clearly identifiable as well. To our knowledge, this is the first complete reproduction of an entire set of experimental data (including both fragment size distribution observables and kinematic observables) by means of a microcanonical multifragmentation model. Moreover, it was shown for the first time that the parameters of the experimentally obtained equilibrated source can be uniquely determined by means of such a model.

\section{Acknowledgments}

The authors acknowledge the support of the Alexander von Humboldt Foundation during this work.
[1] D. H. E. Gross, Rep. Progr. Phys. 53, 605 (1990).

[2] J. P. Bondorf, A. S. Botvina, A. S. Iljinov, I. N. Mishustin and K. Sneppen, Phys. Rep. 257, 133 (1995).

[3] J. Randrup and S. Koonin, Nucl. Phys. A471, 355c (1987).

[4] Al. H. Raduta and Ad. R. Raduta, Phys. Rev. C 55, 1344 (1997).
[5] Al. H. Raduta and Ad. R. Raduta, Phys. Rev. C 61, 034611 (2000).

[6] J. D. Frankland et al., Nucl. Phys. A689, 905 (2001).

[7] J. D. Frankland et al., Nucl. Phys. A689, 940 (2001).

[8] A. S. Iljinov et al., Nucl. Phys. A543, 517 (1992).

[9] J. Randrup, Comput. Phys. Commun. 59, 439 (1990).

[10] Al. H. Raduta, Nucl. Phys. A683, 618 (2001). 
[11] D. H. E. Gross, Phys. Rep. 279, 119 (1997).

[12] G. Fai and J. Randrup, Nucl. Phys. A381, 557 (1982).

[13] G. Tabacaru et al., Proceedings of "Structure of the nucleus at the dawn of the century", Bologna, Italy, 2000, World Scientific, in press, nucl-ex/0102015.

[14] For each particular fragmentation event the origin of the system is considered in its center of mass and not in the center of the spherical "recipient" frame. Therefore, at each step of the Metropolis type simulation one has to consider the transformation $\mathbf{r}_{i}=\mathbf{r}_{0}^{i}-\mathbf{r}_{\mathrm{c} . \mathrm{m} .}, i=1, \ldots, N$.
Here $\mathbf{r}_{0}^{i}, i=1, \ldots, N$ represent the positions of the fragments subject to the origin of the spherical recipient, generated as in [4].

[15] For a $N$ fragment partition the Wigner Seitz Coulomb interaction energy writes: $V_{W S}=\frac{3}{5}\left(\frac{Z^{2}}{R}-\sum_{i=1}^{N} \frac{Z_{i}^{2}}{R_{C}^{i}}\right) e^{2}$ where $R$ is the radius of the freeze-out recipient and $R_{C}^{i}$ is the radius of the Wigner Seitz cell corresponding to the fragment $i, R_{C}^{i}=r_{0}\left(n A_{i}\right)^{1 / 3}$ where $r_{0}=1.2 \mathrm{fm}$, and $n=V / V_{0}$. 